\title{
Michel Chodkiewicz (1929-2020)
}

\author{
Denis Gril \\ Aix-Marseille Univ, CNRs, IRE MAM, Aix-en-Provence, France \\ gril.denis@gmail.com
}

Le 31 mars 2020, Michel Chodkiewicz nous a quitté à l'âge de 90 ans. Il ne publiait ni ne participait plus à des colloques depuis un certain nombre d'années, mais se tenait au courant des publications et continuait à entretenir une correspondance importante avant que la maladie ne finisse par limiter son activité.

C'est un grand spécialiste du soufisme et de l'œuvre du grand maître andalou Ibn al-'Arabì (m. 638/1240) en particulier, que nous venons de perdre. Son parcours dans le domaine des études arabes et islamiques est on ne peut plus atypique car Michel Chodkiewicz ne rejoint l'Université qu'à la toute fin de sa carrière. Il commence dès le début des années cinquante l'étude de l'arabe et de l'islam et s'intéresse très tôt au soufisme et à Ibn al-Arabī dont il ne cesse, tout au long de sa vie, de lire et de méditer l'œuvre immense. Mais c'est dans l'édition qu'il fait carrière. Entré jeune aux éditions du Seuil à Paris, il en gravit tous les échelons jusqu'à en devenir le Président Directeur Général de 1979 à 1989. Son métier d'éditeur le met en contact avec d'éminents universitaires, spécialistes de sciences humaines, historiens en particulier, qui apprécient sa compétence éditoriale, sa vaste culture et sa profonde connaissance de l'islam et de sa spiritualité. Dès 1982, il se voit confier une charge de conférence à l'EHESS puis, en 1989, ayant pris sa retraite des éditions du Seuil, il est recruté comme Directeur d'Études dans cette même institution et y assure un cours intitulé « Histoire de la sainteté dans les sociétés musulmanes arabophones ». Il prend sa retraite définitive en 1994, tout en continuant à participer à des rencontres scientifiques et à publier puis se retire progressivement de la vie publique. En tant qu'éditeur puis enseignant-chercheur, Michel Chodkiewicz voyage et participe à un grand nombre de colloques et de conférences en France et de par le monde. Outre son intérêt majeur pour l'œuvre d'Ibn al-Arabī, il s'implique activement dans plusieurs groupes de recherche sur les voies soufies et sur la sainteté en islam, souvent dans une perspective comparatiste. Il tisse également un vaste réseau de connaissances au cours de ses 
voyages, de rencontres scientifiques et surtout grâce à une abondante activité épistolaire. Il partageait son vaste savoir avec une générosité et une diligence qui impressionnaient ses correspondants.

Son œuvre, comme le montre sa bibliographie jointe en annexe, se partage entre ses livres, traduits pour la plupart en anglais et dans d'autres langues, ses articles consacrés à Ibn al-'Arabī et ceux ayant trait à l'histoire de la sainteté et du soufisme.

Son premier ouvrage Emir Abd el-Kader, Écrits spirituels (1982), traduction de chapitres des Mawāqif, fait connaître la dimension spirituelle de l'Émir et de son œuvre, peu connue jusqu'alors. Une importante introduction ouvre le dossier de la transmission de l'œuvre d'Ibn al-Arabī qui sera repris par la suite. Le choix de ces textes, inspirés par le Coran et l'orientation la plus métaphysique de la doctrine d'Ibn al-'Arabī, constitue une première introduction à cette dernière. C'est pour dissiper quelques malentendus sur la doctrine de l'unité de l'Être (wahdat al-wuğūd) que Michel Chodkiewicz introduit et retraduit en 1982 l'Épître sur l'Unicité absolue d'Awḥad al-Dīn al-Balyānī (m. 686/1288), précédemment attribuée à Ibn al-'Arabī. Son auteur, influencé par Ibn Sabiin (m. 668 ou 669/1269-1271), y soutient le caractère purement illusoire du monde, alors qu'Ibn al-'Arabī considère celui-ci comme le lieu de manifestation des théophanies divines. Cette épître se présentant comme un commentaire de la tradition "Qui se connaît soi-même connaît son Seigneur », l'introduction montre ce qui différencie la doctrine de la connaissance de cet auteur de celle d'Ibn al-'Arabī. Michel Chodkiewicz a été ensuite le maitre d'œuvre des Illuminations de la Mecque (1989), anthologie de traductions d'extraits d'al-Futūhāt al-Makkiyya. Il a surtout écrit une importante introduction, premier guide de lecture de l'œuvre principale d'Ibn al-'Arabī. C'est sans doute Le Sceau des saints : prophétie et sainteté dans la doctrine d'Ibn Arabî (1986) qui a connu la diffusion la plus large. Michel Chodkiewicz y expose quelques-unes des notions clés de la doctrine d'Ibn al-'Arabī, telles al-insān al-kāmil et al-haqīqa l-muhammadiyya, et leurs fondements métaphysiques et leurs implications hagiologiques, tout en inscrivant cette doctrine dans l'histoire du soufisme et de la spiritualité en islam. Un océan sans rivage :Ibn Arabî, le Livre et la Loi (1992), comme l'indique le sous-titre, démontre la relation essentielle et structurelle entre l'œuvre d'Ibn al-'Arabī et le Coran. Si l'abondance des références au Coran et au hadith n'échappe pas à tout lecteur, des Futūhāt en particulier, il fallait attendre Un océan sans rivage pour saisir la relation entre sa composition - et celle d'autres ouvres - et l'ordonnance du Coran. La Révélation instaurant la relation entre le Seigneur et le serviteur, c'est par la conformité à la Loi que l'homme réalise sa perfection. Par ces études, Michel Chodkiewicz, sans couvrir certes tous les champs possibles des études sur le 
Šayh al-akbar leur a donné néanmoins une impulsion décisive en les réorientant vers la source coranique et prophétique de son inspiration.

Plusieurs de ses articles abordent des aspects particuliers de la doctrine et de l'œuvre d'Ibn al-'Arabī, d'autres ouvrent un vaste champ de recherche qui ne cesse de se développer : ses continuateurs, ses opposants et la diffusion de son œuvre. Son étude «Maître Eckhardt et Ibn Arabî » mérite une mention spéciale. Tout en se montrant très réservé sur les rapprochements souvent peu scientifiques entre auteurs de traditions et d'époques différentes, Michel Chodkiewicz se livre ici à une approche doctrinale comparée dont la rigueur est exemplaire et convaincante.

Ses articles sur l'histoire du soufisme traitent de deux domaines différents et complémentaires : d'un côté, les modalités et les typologies de la sainteté, émanant souvent de rencontre à caractère comparatiste entre le christianisme et l'islam ; de l'autre, l'histoire des voies spirituelles (țuruq) et les modes de transmission initiatique, dans le cadre de recherches collectives sur les confréries en islam. De la première série, on retiendra « Le modèle prophétique de la sainteté en islam » (1994) qui fait le lien entre prophétie et sainteté, en continuité avec Le Sceau des saints. « La sainteté et les saints en islam » (1995) inscrit dans la longue durée le statut de la sainteté dans l'histoire doctrinale. «Les quatre morts du soufi » (1998) sur la mort et la renaissance initiatiques ou «la sainteté féminine dans l'hagiographie historique ( (1995) ou encore «La somme des miracles des saints de Nabhânî » (2000) répondent chacun à la volonté de souligner la spécificité de l'hagiologie islamique tout en fournissant des éléments de comparaison avec le christianisme. Sollicité pour conclure les actes d'une rencontre sur les hommes de Dieu dans l'islam et le christianisme, Michel Chodkiewicz, dans «Quelques leçons du comparatisme» (2003) concluait prudemment et positivement: «Mais le profit le plus certain que chaque spécialiste peut retirer de cet échange amical, c'est sans doute le regard neuf qu'il le conduit à porter sur son propre champ d'étude dans ce qu'il a d'unique ». Michel Chodkiewicz reste pour nous un modèle de rigueur et d'ouverture, de réserve et de générosité scientifique.

\section{Bibliographie de Michel Chodkiewicz ${ }^{1}$}

\section{Ouvrages}

'Abd al-Qādir al-Ǧazāinīī, Émir Abd el-Kader : écrits spirituels, présentés et traduits de l'arabe par Michel Chodkiewicz, Paris, Éditions du Seuil, 1982.

1 L'essentiel de cette bibliographie a été établi par Claude Addas. 
Traduction anglaise: The Spiritual Writings of Amir Abd al-kader, trad. James Chrestensen et Tom Manning, Albany, State University of New York Press (« Suny Series in Western Esoteric Traditions »), 1995.

Traduction italienne : Il Libro delle soste, trad. Anna Silva, Milan, Rusconi ( « Testi di spiritualità »), 1984; Milan, Bompani, 2001; Milan, Luni Editrice («Grandi pensatori d'Oriente e d'Occidente », 54), 2016.

Awḥad al-Dīn Balyānī, Épître sur l'Unicité Absolue, présentation et traduction par Michel Chodkiewicz, Paris, Les Deux Océans, 1982.

Michel Chodkiewicz, Le Sceau des saints : prophétie et sainteté dans la doctrine d'Ibn Arabî, Paris, Gallimard («Bibliothèque des sciences humaines », 86), 1986 ; édition revue et augmentée, Paris, Gallimard («Collection Tel », 394), 2012.

Traduction anglaise: Seal of the Saints: Prophethood and Sainthood in the Doctrine of Ibn 'Arabī, trad. Liadain Sherrard, Cambridge, The Islamic Texts Society («Golden Palm Series »), 1993.

Traduction arabe : al-Walāya wa-l-nubuwwa inda l-Šayh Muhyȳ l-Dīn b. al-'Arabī, trad. Aḥmad al-Ṭayyib, Marrakech, Dār al-qubba l-zarqā’ ${ }_{1999^{1}}$; al-Walāya, trad. Aḥmad al-Ṭayyib, Le Caire, al-Mağlis al-a'lā li-l-țaqāfa, 2000² .

Traduction italienne: Il Sigillo dei santi : Profezia e santità nella dottrina di Ibn 'Arab̂̀, trad. Guido Mongini, Brescia, Morcelliana («Scienze e storia delle religioni. Nuova serie », 10), 2009.

Ibn 'Arabī, Les Illuminations de La Mecque :textes choisis, dir. et trad. Michel Chodkiewicz avec la collaboration de Cyrille Chodkiewicz et Denis Gril, Paris, Sindbad ( La Bibliothèque de l'Islam. Textes»), 1989; Paris, Albin Michel («Spiritualités vivantes », 150), 1997 .

Michel Chodkiewicz, Un océan sans rivage : Ibn Arabî, le Livre et la Loi, Paris, Éditions du Seuil (« La librairie du XX ${ }^{\mathrm{e}}$ siècle »), 1992.

Traduction anglaise: An Ocean without Shore: Ibn Arabi, the Book and the Law, trad. David Streight, Albany, State University of New York Press, 1993.

Traduction arabe : Baḥr bi-lā sāḥil, trad. Aḥmad al-Ṣādiqī, Beyrouth, Dār al-manār al-islāmī, 2018.

Traduction turque: Sahilsiz bir umman: Hakikat, Seriat ve Ibn Arabî, trad. Atilla Ataman, Istanbul, Gelenek Yayıncılık, 2003 ; Istanbul, Nefes Yayinlari, 2015.

\section{Articles sur l'histoire du soufisme}

Michel Chodkiewicz, «Les secrets de la futuwwa», dans Proche-Orient et Tiers-monde, 7 (juin 1983) [Présence de l'islam, dir. Charles Saint-Prot], p. 49-56.

Id., «Quelques aspects des techniques spirituelles dans la Tarîqa Naqshbandiyya », dans Naqshbandis: cheminements et situation actuelle d'un ordre mystique musulman: actes de la table ronde de Sèvres 2-4 mai 1985, éds Thierry Zarcone, Marc 
Gaborieau et Alexandre Popovic, Istanbul-Paris, Isis («Varia Turcica », 18), 1990, p. 69-82.

Id., «Le saint illettré dans l'hagiographie islamique », Cahiers du centre de recherches historiques, 9 (avril 1992), p. 31-41.

Id., «Le modèle prophétique de la sainteté en islam », Al-Masâq, 7 (1994), p. 201-226.

Id., «La réception du soufisme par l'occident: conjectures et certitudes », dans The

Introduction of Arabic Philosophy into Europe, éds Charles E. Butterworth et Blake Andrée Kessel, Leyde-New York-Cologne, E.J. Brill («Studien und Texte zur Geistesgeschichte des Mittelalters », 39), 1994, p. 136-149. [version révisée de la communication présentée à l'Université euro-arabe, Bologne, 1988]

Id., « La sainteté et les saints en islam », dans Le culte des saints dans le monde musulman, dir. Henri Chambert-Loir et Claude Guillot, Paris, Publication de l'école française d'Extrême Orient («Études thématiques », 4), 1995, p. 13-32.

$I d$., «La sainteté féminine dans l'hagiographie islamique », dans Saints orientaux, dir.

Denise Aigle, Paris, De Boccard («Hagiographies médiévales comparées », 1), 1995, p. 99-115.

Id., « Rūḥāniyya », EI $I^{2}$ (1995).

Id., «Le soufisme au XXI" siècle», dans Les Voies d'Allâh: les ordres mystiques dans l'islam des origines à aujourd'hui, dir. Alexandre Popovic et Gilles Veinstein, Paris, Fayard, 1996, p. 532-543.

Id., « Les quatre morts du soufi », Revue de l'histoire des religions, 215/1 (1998), p. 35-57. Id., « Les maîtres spirituels en islam », Connaissance des religions, 53-54 (1998), p. 33-48. Id., « Note complémentaire sur les rites d'initiation dans les turuq », 'Ayn al-hayât, Quaderno du Studi della Tarîqa Naqshbandiyya, 5 (1999), p. 45-64; republié sous le titre «Les rites initiatiques dans le soufisme », Connaissance des religions, 69-70 (2003), p. 81-95.

Id., «La somme des miracles des saints de Nabhânî », dans Miracle et Karāma, dir. Denise Aigle, Turnhout, Brepols («Hagiographies médiévales comparées», 2; «Bibliothèque de l'École des hautes études. Sciences religieuses», 109), 2000, p. $607-622$.

Id., « Les musulmans et la Parole de Dieu », Revue de l'histoire des religions, 218/1 (2001), p. 13-31.

Id., « Nous leur montrerons Nos signes ...», dans Stigmates, dir. Dominique de Courcelles, Paris, Éditions de l'Herne (« Cahiers de l'Herne », 75), 2001, p. 37-42.

Traduction anglaise : «We will show them our signs ... », Journal of the Muhyiddin Ibn Arabi Society, 50 (2011), p. 23-33.

Id., «Quelques leçons du comparatisme», dans Histoire des hommes de Dieu dans l'islam et le christianisme, dir. Dominique Iogna-Prat et Gilles Veinstein, Paris, Flammarion, 2003, p. 279-286. 


\section{Articles concernant Ibn al-'Arabì et l'école akbarienne}

Id., «Ibn 'Arabî, la Lettre et la Loi », dans Actes du colloque Mystique, culture et société,

Université de Paris Sorbonne, éd. Michel Meslin, Paris, Université de Paris-Sorbonne, 1983, p. 27-40.

Id., «Une introduction à la lecture des Futûhât Makkiyya», dans Les Illuminations de La Mecque : textes choisis, dir. et trad. Michel Chodkiewicz avec la collaboration de Cyrille Chodkiewicz et Denis Gril, Paris, Sindbad ( «La Bibliothèque de l'Islam. Textes »), 1989, p. 19-73.

Id., «L'offrande au Prophète de Muhammad al-Burhânpûrî», Connaissance des Religions, 4/1-2 (1988), p. 30-40.

Id., « Le Coran dans l'œuvre d'Ibn 'Arabî / El Coran en la obra de Ibn 'Arabî », dans Los dos Horizontes: textos sobre Ibn al'Arabi: trabajos presentados al Primer Congreso internacional sobre Ibn al-'Arab̄̄, Murcia, 12-14 de noviembre de 1990, éd. Alfonso Carmona Gonzalez, Murcie, Consejería de cultura educación y turismo Editora regional de Murcia («Colección Ibn Al'Arabi. Extra », 2), 1992, p. 133-162.

Id., «The Esoteric Foundations of Political Legitimacy in Ibn 'Arabî », dans Muhyiddin Ibn 'Arabi: A Commemorative Volume, éds Stephen Hirtenstein et Michael Tiernan, Shaftesbury-Rockport, Element, 1993, p. 190-198.

Id., «Quelques remarques sur la diffusion de l'enseignement d'Ibn ‘Arabî », dans Modes de transmission de la culture religieuse en islam, éd. Hassan Elboudrari, Le Caire, IFAO («Textes arabes et études islamiques », 31), 1993, p. 201-224.

Traduction anglaise: «The diffusion of Ibn al-Arabi's Doctrine», Journal of the Muhyiddin Ibn Arabi Society, 9 (1991), p. 36-57.

Id., « Du devoir d'exil », Le Cheval de Troie, 10 (1994), p. 71-78.

Id., « Un traité yéménite sur l'unicité de l'être », Ayn al-hayât, Quaderno du Studi della Tarîqa Naqshbandiyya, 1 (1995), p. 13-29 ; repris dans Horizons Maghrébins, 30 (1996), p. 81-9o.

Id., « Le voyage sans fin », dans Le voyage initiatique en terre d'islam: ascensions célestes et itinéraires spirituels, dir. Mohammad Ali Amir-Moezzi, Louvain-Paris, Peeters ( Bibliothèque de l'École des hautes études. Section des sciences religieuses », 103), 1996, p. 239-25o.

Traduction anglaise : «The endless voyage »,Journal of the Muhyiddin Ibn Arabi Society, 19 (1996) [The Journey of the Heart, éd. John Mercer], p. 71-84.

Id., « The banner of praise », Journal of the Muhyiddin Ibn Arabi Society, 21 (1997)

[Praise, éd. Stephen Hirtenstein], p. 45-58.

Id., « Les Malâmiyya dans la doctrine d'Ibn Arabî », dans Melâmis/Bayrâmis :études sur trois mouvements mystiques musulmans, éds Nathalie Clayer, Alexandre Popovic et Thierry Zarcone, Istanbul, Isis, 1998, p. 15-25.

Id., «Le procès posthume d'Ibn 'Arabî », dans Islamic Mysticism Contested: Thirteen Centuries of Controversies and Polemics, éds Frederick de Jong et Bernd Radtke, Leyde-Boston, Brill («Islamic History and Civilization », 29), 1999, p. 93-123. 
Id., «The Futûhât al-makkiyya and its Commentators: Some Unresolved Enigmas », dans The Heritage of Sufism, II, The Legacy of Medieval Persian Sufism (1150-1450), ed. Leonard Lewisohn, Oxford-Boston, Oneworld, 1999, p. 219-232.

Id., « Maître Eckhart et Ibn Arabî », Mémoire Dominicaine, 15 (2001), p. 21-36.

Id., «Les trois cailloux du shaykh 'Abd al-Karîm al-Jîlı̂», dans Mystique musulmane: parcours en compagnie d'un chercheur, Roger Deladrière, éd. Geneviève Gobillot, Paris, Cariscript («Études chrétiennes arabes », 21), 2002, p. 141-154.

Id., « La vision de Dieu selon Ibn Arabî », dans Autour du regard: Mélanges Gimaret, éd. Éric Chaumont, Louvain, Peeters, 2003, p. 159-173.

Traduction anglaise: «The vision of God according to Ibn 'Arabi », Journal of the Muhyiddin Ibn Arabi Society, 14 (1993) [Prayer and Contemplation, éd. Stephen Hirtenstein], p. 53-67.

Id., «Shaykh Muhammad Demirdâsh : un soufi akbarien au XVI ème siècle », Horizons Maghrébins, $5^{1}$ (2004), p. 20-39.

Id., «Ibn Arabî dans l'œuvre d'Henri Corbin », dans Henri Corbin: philosophies et sagesses des religions du livre, éds Mohammad Ali Amir-Moezzi, Christian Jambet et Pierre Lory, Turnhout, Brepols («Bibliothèque de l'École des hautes études. Sciences religieuses », 126 ; « Histoire et prosopographie de la section des sciences religieuses », 1), 2005, p. 81-91.

Id., « Le paradoxe de la Ka'ba », Revue de l'histoire des Religions, 222 (2005), p. 435-461.

Traduction anglaise : «The Paradox of the Ka'ba », Journal of the Muhyiddin Ibn Arabi Society, 57 (2015), p. 57-83.

Id., « La réception de la doctrine d'Ibn Arabî dans le monde ottoman », in Sufism and Sufis in Ottoman Society, éd. Ahmet Yaşar Ocak, Ankara, Atatürk Supreme Council for Culture, Language and History («Publications of the Turkish Historical Society. XXX. serial», 3), 2005, p. 97-120.

Id., «Mi'râj al-kalima: de la Risâla Qushayriyya aux Futûhât makkiyya», dans Reason and Inspiration in Islam: Theology, Philosophy and Mysticism in Muslim Thought: Essays in Honour of Hermann Landolt, ed. Todd Lawson, Londres, I.B. Tauris en association avec l'Institute of Ismaili Studies, 2005, p. 248-261.

Traduction anglaise : «Mirâj al-kalima: From the Risâla Qushayriyya to the Futûhât makkiyya », Journal of the Muhyiddin Ibn Arabi Society, 45 (2009), p. 1-19.

Id. et Claude Addas, «On Two Books Attributed to Ibn 'Arabi : Kitāb al-mabādī wa l-ghāyāt li-ma'ānì l-hurūf and Kitāb māhiyyat al-qalb», Journal of the Muhyiddin Ibn Arabi Society, 62 (2017), p. 105-114.

\section{Divers}

Michel Chodkiewicz, «Éditer la littérature arabe traduite», Rencontre autour de la littérature romanesque égyptienne traduite en français, Le Caire 15-17 octobre 199o, éd. Sayyed al-Bahrawi et al., Le Caire, Université du Caire-Ambassade de France au Caire, 1992, p. 131-138. 


\section{Préfaces d'ouvrages}

Rachida Chih, Le soufisme au quotidien: confréries d'Égypte au XXe siècle, Paris-Arles, Sindbad-Actes Sud («Bibliothèque arabe. Collection Hommes et sociétés »), 2000. Éric Geoffroy, Le soufisme en Égypte et en Syrie sous les derniers Mamelouks et les premiers Ottomans : orientations spirituelles et enjeux culturels, Damas, Institut Français de Damas, 1995.

Sitt 'Ağam bt al-Nafīs b. al-Qāsim b. Țuraz al-Bag̉dādiyya, Šarḥ al-mašāhid al-qudsiyya li-takmül dä̉irat al-ḩatm al-mawșūf bi-l-walāya l-muhammadiyya Muhammad $b$. al-'Arabī, éds Bakrī 'Alā’ al-Dīn et Su'ād al-Hakīm, Damas, Institut français du Proche Orient, 2004.

Christian Bonaud, Le soufisme: "al-tașawwuf" et la spiritualité islamique, Paris, Maisonneuve et Larose-Institut du Monde Arabe («Collection Islam-Occident», 8), 1991.

Muhyī l-Dīn b. 'Arabī, A prayer for Spiritual Elevation and Protection: al-Dawr al-a $\overline{l a ̄}$ (Hizb al-wiqāya), trad. Suha Taji-Farouki, Oxford, Anqa, 2012.

Marie-France Mourrégot, L'islam à l'île de la Réunion, Paris, L'Harmattan, 2010.

À parâtre en anglais : préface à l'édition critique des Mawāqif de l'Émir Abd el-Kader, Journal of the Muhyiddin Ibn Arabi Society.

\section{Comptes rendus}

48 comptes rendus dans les Studia Islamica de 1983 à 2003.

29 comptes rendus dans le Bulletin Critique des Annales Islamologiques de 1984 à 2003. 31 comptes rendus dans L'Histoire de 1985 à 1996.

Maḥmūd Ġurāb et Michel Chodkiewicz, « Maḥmūd Ghurāb : "Sharḥ Fuṣūṣ al-Ḥikam” », Studia Islamica, 76 (1992), p. 177-180².

2 Cet article, paru dans la rubrique « Notes et commentaires» des Studia Islamica, comprend une réponse de Maḥmūd Gurāb à une recension de son Šarh Fuṣūs al-ḥikam par Michel Chodkiewicz, suivi d'une lettre de réponse de ce dernier à l'auteur de l'ouvrage recensé. 